\title{
Controlling Walking Behavior of Passive Dynamic Walker utilizing Passive Joint Compliance
}

\author{
Takashi TAKUMA, Koh HOSODA \\ Department of Adaptive Machine Systems, Graduate School of Engineering \\ Osaka University \\ Yamadaoka 2-1, suita, Osaka, Japan \\ \{takuma,hosoda\}@ams.eng.osaka-u.ac.jp
}

\begin{abstract}
The passive dynamic walker (PDW) has a remarkable characteristic that it realizes cyclic locomotion without planning the joint trajectories. However, it cannot control the walking behavior because it is dominated by the fixed body dynamics. Observing the human cyclic locomotion emerged by elastic muscles, we add the compliant hip joint on PDW, and we propose a "phasic dynamics tuner" that changes the body dynamics by tuning the joint compliance in order to control the walking behavior. The joint compliance is obtained by driving the joint utilizing antagonistic and agonistic McKibben pneumatic actuators. This paper shows that PDW with the compliant joint and the phasic dynamics tuner enhances the walking performance than present PDW with passive free joints. The phasic dynamics tuner can change the walking velocity by tuning the joint compliance. Experimental results show the effectiveness of the joint compliance and the phasic dynamics tuner.
\end{abstract}

\section{INTRODUCTION}

One of the interesting characteristics of human locomotion is that they realize stable cyclic locomotion. Cyclic locomotion is obtained by phasic actuation [1] on the passive joints, and it does not require planning the joint trajectories. On the other hand, present bipedal walkers plan joint trajectories based on some criteria such as Zero Moment Point [2] or an inverted pendulum model [3]. Although the bipedal walkers realize stable locomotion utilizing such approaches, they require precise models of robot dynamics and environment to calculate the joint trajectories.

The passive dynamic walker (PDW) also realizes cyclic locomotion without planning the joint trajectories [4]. PDW walks on shallow slope utilizing its body dynamics and the interaction with the terrain. It also walks on flat plane with simple actuation [5]. However, it cannot change its walking behavior because the behavior is dominated by body dynamics, and each dynamics of the present PDW is fixed. Observing human locomotion, it seems that they change their dynamics by tuning the joint compliance to control control the walking. In [6], though it is case of running, the hip joint compliance is changed according to desired stride frequency. Although some researches on bipedal walkers have also reported the importance of compliant joints [7], [8], there is no report that shows a contribution of the joint compliance by the physical bipedal walker to the best of our knowledge.

In this paper, the hip joint compliance is focused to realize enhanced walking performance of the PDW. We propose a phasic dynamics tuner to change the robot dynamics by tuning the joint compliance. By utilizing the phasic dynamics tuner, this paper shows experiment results that the walking velocity is changed by tuning the joint compliance. This result shows that the joint compliance contributes to enhance the walking performance of the PDW.

In order to change the hip joint compliance, the McKibben pneumatic actuator [9], [10] is adopted. The McKibben pneumatic actuator has elasticity and that can be changed according to the inner pressure. We set the actuators on agonistic and antagonistic position around the joint in order to change joint compliance as well as actuation. The actuator is driven by opening supply / expel valves, and the phasic dynamics tuner is realized by determining the duration to open / close supply / expel valves. In order to show the contribution of the joint compliance, we have experiments to observe the walking performance when the robot walks with passive free hip joint and when the robot walks with passive compliant joint.

\section{A 2D Bipedal WAlKer With PNEUMatic ACTUATORS}

\section{A. Joint mechanism driven by pneumatic actuators}

It is expected that passive compliance of the robot joint contributes to operate a walking behavior, walking velocity in this paper, of the passive walker. In order to realize the passive hip joint compliance, it is effective to adopt the joint mechanism driven by the agonistic and antagonistic McKibben pneumatic actuators [7]. Figure 1 shows the architecture of a planar bipedal walker driven by the antagonistic pneumatic actuators. When the air is supplied into the actuator, the actuator shrinks, generating tensile force. The actuator has a nonlinear springy nature, and the stiffness increases according to the increase in the pressure. The joint compliance is therefore determined by the pressure of the actuators.

Three-position solenoid valves are adopted to operate the pressure. The valve switches three states: supplying air from the source, expelling air to the atmosphere, and closing valves to quit both supplying and expelling. Figure 2 shows a pressure profile when the compressed air $(0.6 \mathrm{MPa})$ is supplied into the actuator. The solid line shows the profile when the supply valve is always open. It takes about $0.8 \mathrm{~s}$ to reach $0.6 \mathrm{MPa}$. Considering that the supply / expel valve can be closed at arbitral time and that the response time to open / close valve 


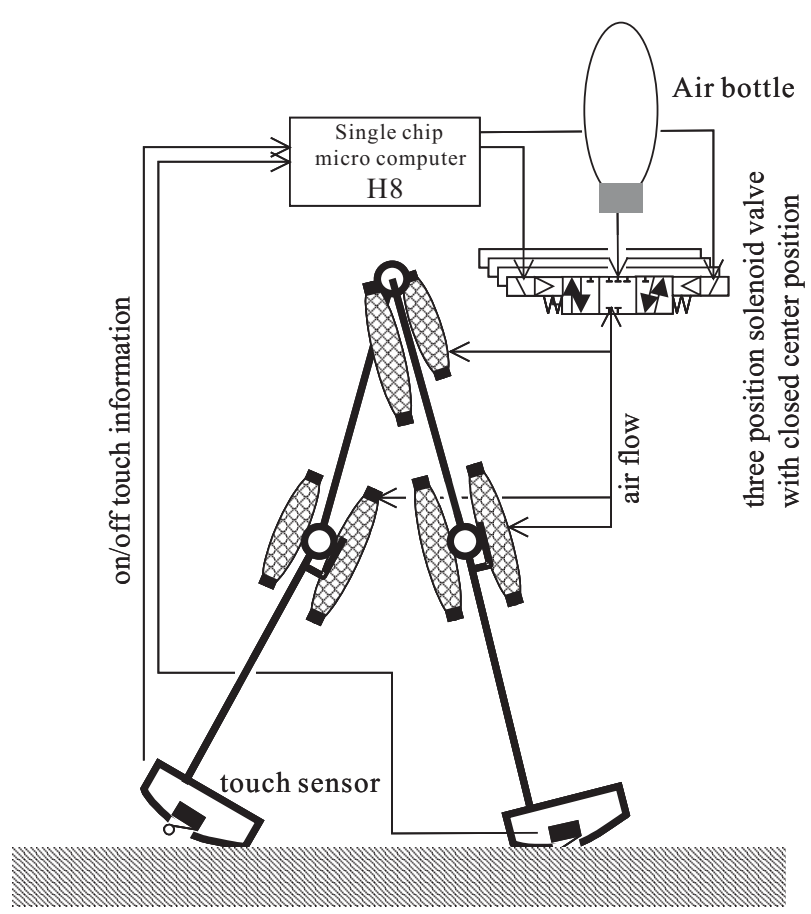

Fig. 1. Architecture of a planar bipedal walker driven by antagonistic McKibben pneumatic actuators. The main feature of the walker is the threeposition solenoid valve with a closed center position that enables the robot to control joint compliance.

is less than $0.03 \mathrm{~s}$, it is possible to stop supplying / expelling before the air is completely fulfilled or expelled. By tuning the duration to open the valve, it is possible to realize continuous pressure change. Broken lines in Figure 2 show the pressure profile when the valve is open for certain period $(0.1,0.15$, and $0.2 \mathrm{~s}$ ) and then close. When the duration is shorter, the pressure after closing the valve is lower and vice versa. Because joint compliance is changed according to the pressure, joint compliance is then controlled by tuning the durations to open supply / expel valve. The difference between [11] is that [11] adopts a two-position solenoid valve that either supplies or expels. Therefore, it cannot maintain certain compliance of the joint.

\section{B. Specification}

Figure 3 shows real-world prototype "Que-kaku". All of the joints are driven by antagonistic McKibben pneumatic actuators [9], [10] by HITACHI Medical Corporation [12]. The length and radius (in contraction) of the actuators are $0.15 \mathrm{~m}$ and $0.027 \mathrm{~m}$, respectively. The prototype generates approximately $800 \mathrm{~N}$ when the pressure is $0.7 \mathrm{MPa}$.

The walker has three joints: two knees and one hip. All joints are driven by the antagonistic pneumatic actuators. It is $0.75 \mathrm{~m}$ high, 0.35 wide, and weighs $5.0 \mathrm{~kg}$. Its four legs keep it from swaying side to side. The legs are connected in pairs. The thigh is $0.3 \mathrm{~m}$ long and weighs $2.16 \mathrm{~kg}$, and the shank is $0.35 \mathrm{~m}$ long and weighs $0.48 \mathrm{~kg}$.

The self-contained robot has two $1.2 \mathrm{MPa} \mathrm{CO}_{2}$ bottles weighing $0.7 \mathrm{~kg}$ each with regulators as air sources, a micro-

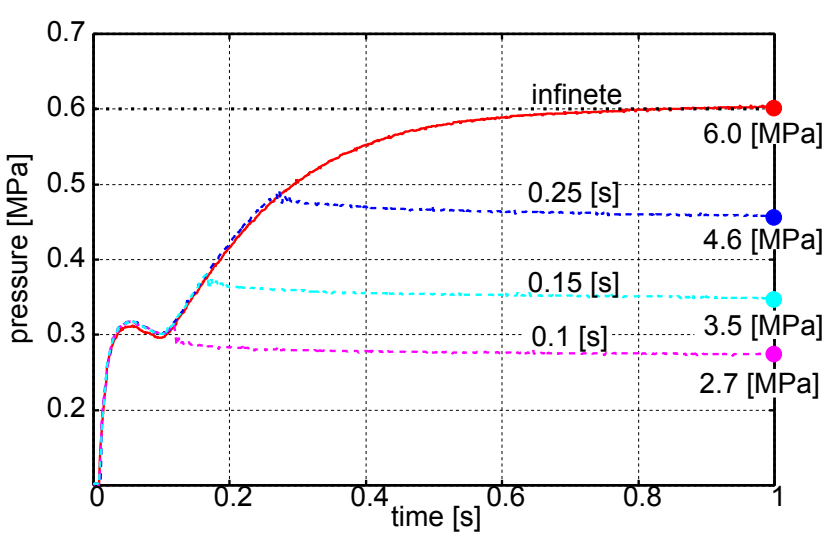

Fig. 2. Pressure profile after opening supply valve. The air of $0.6[\mathrm{MPa}]$ is fulfilled for 0.8 [s] (solid line). The pressure is controlled by tuning the supply duration (broken line : closed at $0.1,0.15$, and $0.25 \mathrm{~s}$ ).

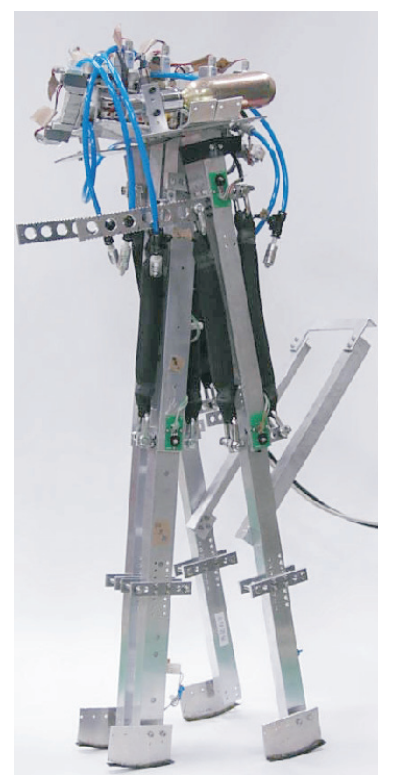

Fig. 3. A 2D bipedal walker driven by McKibben pneumatic actuators. The robot is self-contained and has two air bottles with regulators, three-position air valves, a microcomputer board, and an electrical battery.

computer (H8), and an electrical battery weighing $1.0 \mathrm{~kg}$. The robot's six three-position solenoid valves (SMC Corporation [13] SYJ3340-6M-M5) weigh a total of $0.6 \mathrm{~kg}$. The regulator on each bottle maintains air pressure at $0.55 \mathrm{MPa}$, and valves supply regulated air to muscles. Its feet have round soles with a curvature of $0.125 \mathrm{~m}$ and $0.08 \mathrm{~m}$ long. Its on / off switches detect contact with the ground (Figure 1). Based on on / off information fed to the microcomputer, the computer outputs open and close commands to solenoid valves.

\section{Phasic Dynamics Tuner for Limit Cycles}

It is important for the passive walker to design a robot dynamics including the center of mass and the radius of feet. We designed them by try and error to enable the robot to walk on a flat plane using simple phasic actuation. This paper adopts two feed-forward controller to show the contribution of the 
passive joint compliance on changing walking behavior. One is a simple actuation pattern that the robot walks with a free hip joint, and the other is the pattern that names phasic dynamics tuner such that the robot walks with a passive compliant hip joint. By tuning joint compliance, robot dynamics is controlled, and then it is expected that walking behavior is changed.

\section{A. Feed-forward Controller for walking with the free hip joint}

The valve operation procedure is as follows. The valve opening timing chart is shown in Figure 4:

(i) Begin to swing a leg by opening the supply valve to the agonistic hip actuator. Let the duration to open the supply valve be $T_{s}$.

(ii) In order to swing with passive free hip joint, the expel valve for the agonistic hip actuator is open until landing. The expel valve for the antagonistic hip actuator is always open while walking.

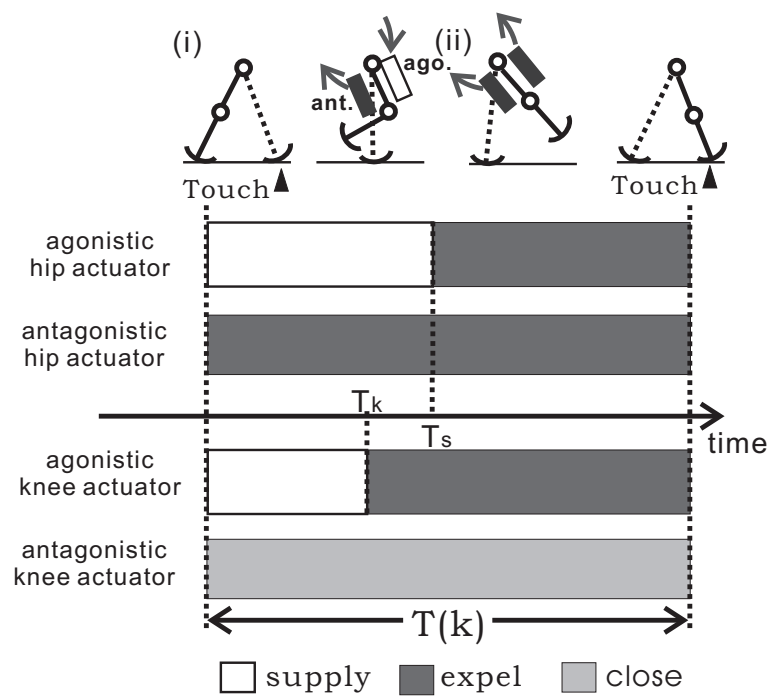

Fig. 4. Feed-forward controller for walking with free hip joint : activating and relaxing muscles. The rectangles in the picture indicate the agonistic and antagonistic hip actuators. Note that the supply duration for the agonistic knee actuator $T_{k}$ is tuned so as to avoid foot collision on the ground.

$T(k)$ shown in the figure is defined as the walking cycle at the $k$ th step.

\section{B. Controller for walking with a passive compliant hip joint (phasic dynamics tuner)}

The valve operation procedure is as follows. The valve opening timing chart is shown in Figure 5:

(i) Begin to swing a leg by opening the supply valve to the agonistic hip actuator. Let the duration to open the supply valve be $T_{s}$. Expel valve to the antagonistic hip actuator is open simultaneously for $T_{e}$.

(ii) Close both supply and expel valves. When the landing signal is fed from on / off switch on the foot, return to (i).
In (ii), the air remains after closing the valves. Mentioned in previous section, pressure is determined by supplying / expelling duration, and then the joint compliance is changed by duration $T_{s}$ and $T_{e}$.

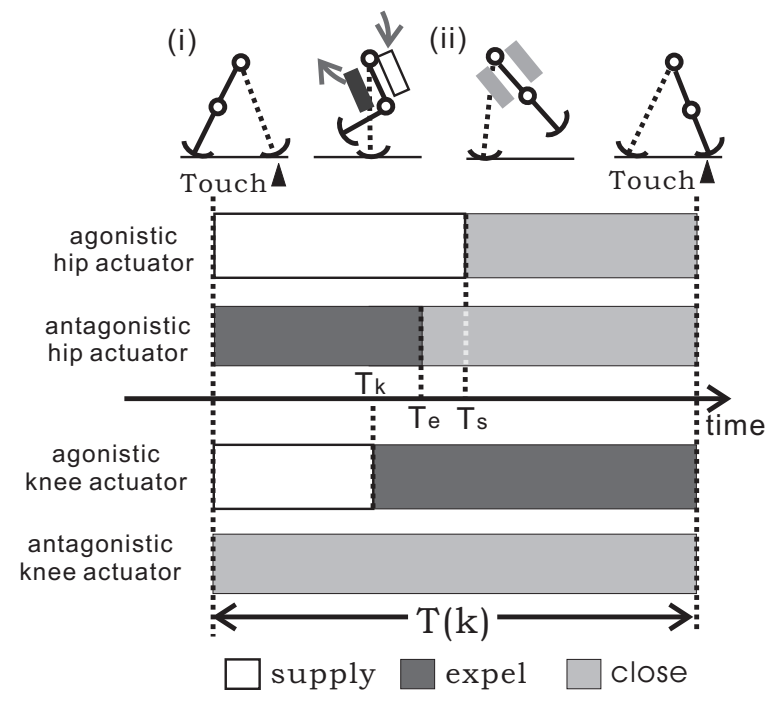

Fig. 5. Timechart for walking with the passive compliant hip joint

For each valve procedure, air is supplied to an antagonistic knee actuator before walking. A knee of support leg keeps extended by closing both valves of agonistic and antagonistic actuators. A knee of swing leg bends by opening supply valve to an agonistic knee actuator for certain period, $T_{k}$ in Figure, and it extends again by opening expel valve to the agonistic knee actuator. We tune $T_{k}$ to avoid the foot collision on the ground. In the preset paper, we fix $T_{k}$ at $0.3 \mathrm{~s}$. Since the movement of the knee is small, it does not strongly affect the behavior.

Pressure profiles of 5 steps on agonistic and antagonistic actuator of the hip joint is shown in Figure 6. Supply duration $T_{s}$ and expel duration $T_{e}$ are $T_{s}=0.2, T_{e}=0.28 \mathrm{~s}$, respectively. The pressure of the agonistic actuator increases due to air supply and the pressure in antagonistic actuator decreases due to air expel. Each pressure keeps almost same after closing the valve, and both actuators co-contract after both valves are closed. Hip joint compliance keeps same without air consumption while closing both valves. The swing leg travels with the passive compliant hip joint.

\section{EXPERIMENT}

\section{A. Experiment setup}

The robot walks on the floor using two types of feedforward controller: controller for walking with free hip joint and for walking with compliant hip joint. An average walking velocity is measured by measuring walking cycle and walking distance of the step. The velocity is measured after 10th step assuming that the walking behavior is converged into the limit cycle after 10 th step.

In both case, the velocity is measured with various walking parameter values. When the robot walks with passive free hip 


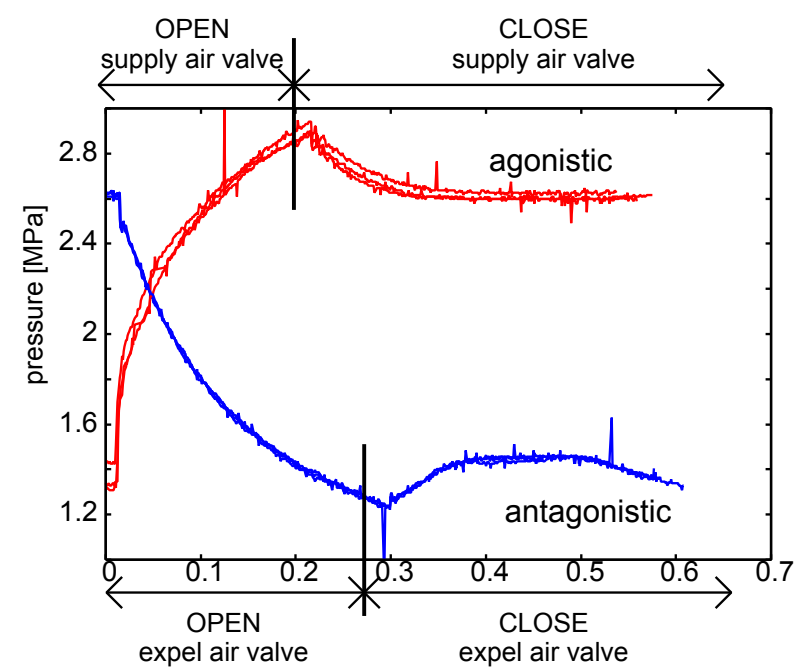

Fig. 6. Pressure profiles of agonistic and antagonistic actuator at one step

joint, the supply duration $T_{s}$, a period of phasic actuation, is changed. When the robot walks with passive compliant hip joint, the expel duration $T_{e}$ of the phasic dynamics tuner is changed. By changing the parameter $T_{e}$ of the tuner, the joint compliance as well as the period of phasic actuation is tuned. $T_{s}$ of the tuner is fixed as $0.2 \mathrm{~s}$ considering that the compliance can be tuned by changing the expel duration and the supply duration is fixed (see Figure 9).

\section{B. Result 1: Walking with a free hip joint}

Figure 7 shows the relationship between supply duration $T_{s}$ and average walking velocity when the robot walks with a passive free hip joint. The velocity and error indicated correspond to average velocity and error of 20 steps at each supply duration. The robot walks when $T_{s}=0.12,0.14$, and $0.16 \mathrm{~s}$. Even though the energy required to swing a leg increases according to longer supply duration, this result shows that the walking parameter does not control the velocity. The walking velocity is dominated by the robot body dynamics. This result shows that the supply duration to swing the leg is not responsible for controlling the velocity and that it is much dominated by the robot body dynamics.

\section{Result 2: Walking with a compliant hip joint}

Figure 8 shows the relation between expel duration $T_{e}$ and the average walking velocity and error. They are obtained from 20 steps at each parameter value. The walking parameter $T_{e}$ is changed from $0.22 \mathrm{~s}$ to $0.4 \mathrm{~s}$. The walking velocity is higher when $T_{e}$ is shorter. This result shows that the walking parameter controls the walking velocity when the robot walks with the proposed phasic dynamics tuner.

\section{Relationship between compliance and velocity}

As shown in the Figure 5, air is supplied to the agonistic actuator of the hip joint and expelled from the antagonistic actuator when the robot walks with the passive compliant hip joint. When the parameter is changed, the pressure profiles of

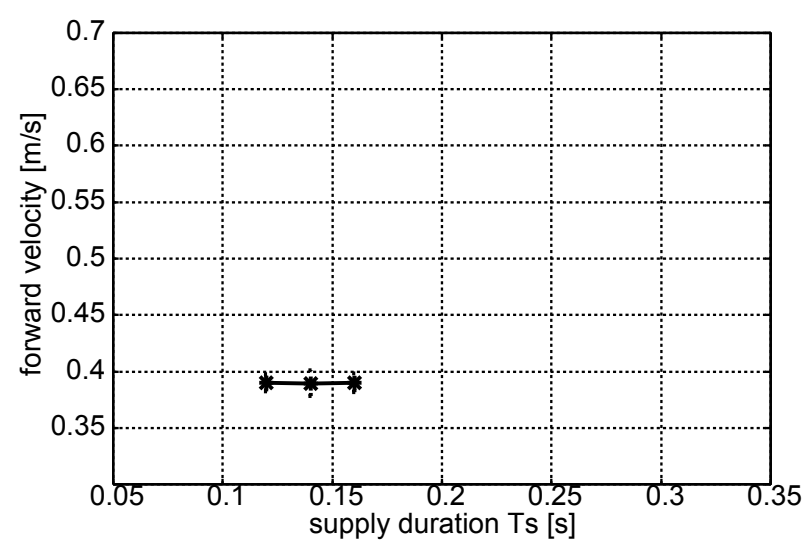

Fig. 7. Relationship between supply duration for the agonistic hip actuator $T_{s}$ and average forward velocity when $T_{s}=0.12,0.14$ and $0.16 \mathrm{~s}$ when the robot walks with a free hip joint

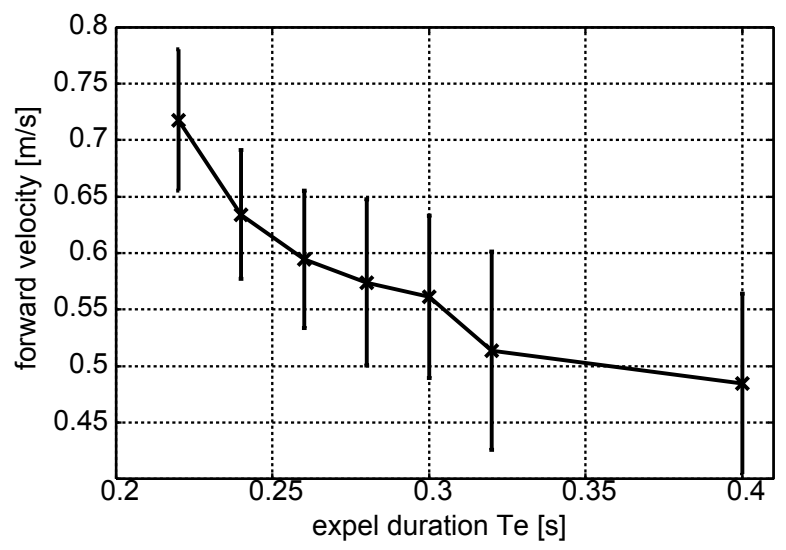

Fig. 8. Relationship between expel duration for antagonistic actuator $T_{e}$ and average forward velocity.

both actuators as shown in Figure 6 are varied. As explained in [10], tensional force of the pneumatic actuator is proportional to pressure. Therefore, considering that the pressures after closing supply and expel valves are almost same and that the hip compliance is determined by both pressure, there is a strong correlation between the pressure at heel strike and hip compliance. We then observe the relationship between the compliance and walking velocity from the relationship between velocity and the pressures of agonistic and antagonistic actuator at heel strike.

Figure 9 shows the relationship between the walking parameter $T_{e}$ and the pressure in the antagonistic (left axis) and agonistic (right axis) actuators. This relationship shows that the pressure at heel strike is changed according to the parameter. When $T_{e}$ is longer, both pressures are lower. It means, considering that the tensional force is smaller when the pressure is lower, that the hip compliance is lower when $T_{e}$ is longer and vice versa.

In order to confirm the relationship between hip joint compliance and velocity, we show the relationship between the pressure of agonistic actuator and velocity by combining above results shown in Figure 8 and in Figure 9. Figure 10 shows 


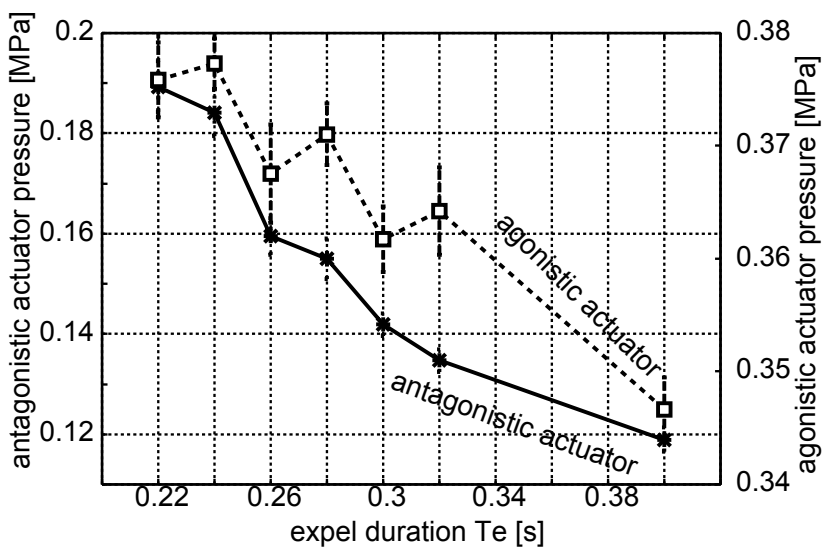

Fig. 9. Relationship between the walking parameter $T_{e}$ and the pressure in the antagonistic actuator at heel strike.

the relationship between pressure of the antagonistic actuator and walking velocity. Considering that the joint compliance is higher when the actuator pressure is higher, this result shows that the walking velocity is higher when hip joint compliance is higher and vice versa. We confirmed similar relationship between pressure of the agonistic actuator and walking velocity. This result shows that the walking velocity is changed by the joint compliance and that the phasic dynamics tuner controls walking behavior of PDW.

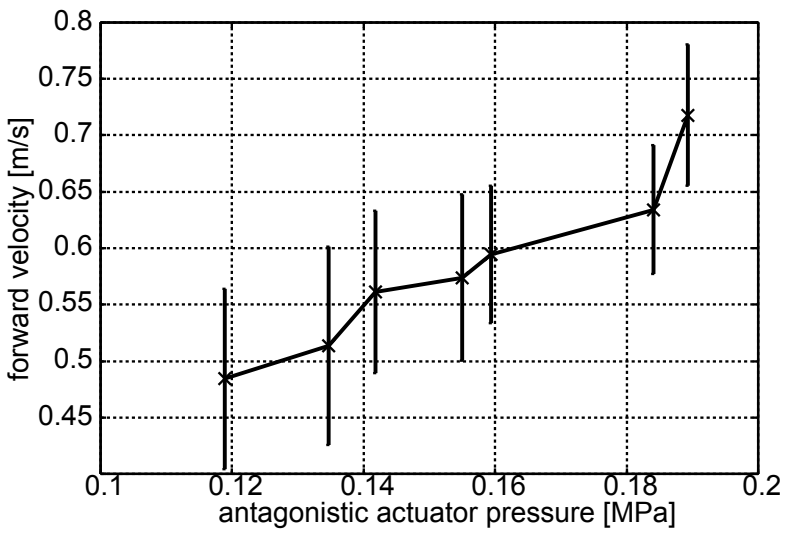

Fig. 10. Relationship between the pressure in the antagonistic actuator at heel strike and the forward velocity.

\section{CONCLUSION AND Discussion}

In this paper, we built real passive walker that has characteristic joint mechanism driven by agonistic and antagonistic McKibben pneumatic actuators. In order to obtain tunable joint compliance, we utilize air valves that switch supplying, expelling, and stopping both supplying and expelling. We showed that the walking behavior, the walking velocity, is changed by tuning the joint compliance.

In order to change the passive hip joint compliance, we used the phasic dynamics tuner that tune the hip joint compliance. By changing the joint compliance, the robot dynamics is changed. We then changed the walking parameter of the tuner and observed the walking velocity. In order to confirm the contribution of the passive compliant hip joint, we used two types of feed-forward controllers. One is for walking with passive free hip joint, and the other is for walking with passive compliant hip joint (phasic dynamics tuner). We compared the walking performance with free and compliant hip joint.

The experimental results showed that the velocity could be controlled by changing walking parameter when the robot walks with passive compliant hip joint. On the other hand, the velocity could not be controlled when the robot walks with passive free hip joint. These results lead us that the velocity could be changed by the phasic dynamics tuner.

It is difficult to analyze waling behavior of the passive dynamic walker with the pneumatic actuators because the actuator has complex non-linearity such as a hysteresis. Therefore, it is difficult to explain why the walking velocity is changed by tuning the joint compliance. The issue to analyze remains to be solved. In order to show the contribution of the compliant joint, we will investigate the effect not only of the hip joint but of another joint. One of the candidates to be investigated is an absorption and a reutilization of the collision impact at heel strike by ankle and knee joint. This effect will play important role not only in case of walking but in case of running.

Lastly, we discuss the relationship between air consumption and the walking velocity. Figure 11 shows the relationship between air consumption ( $l$ per 50 steps) and the expel duration $S_{e}$ while $S_{f}$ is fixed as $0.2 \mathrm{~s}$. Due to the limitation of the experiment space, this result is not observed when the robot walks on the floor explained in previous section but on a treadmill. This result indicates that air consumption is smaller when $S_{e}$ is shorter. Combining this result and the one shown in Figure 8, air consumption is smaller when the walking velocity is higher, $S_{e}$ is shorter, and vice versa. Note that air is consumed for driving the hip joint and knee joint, and it is not corresponding to metabolic cost in human case that includes energy consumption to preserve contracting the muscle.

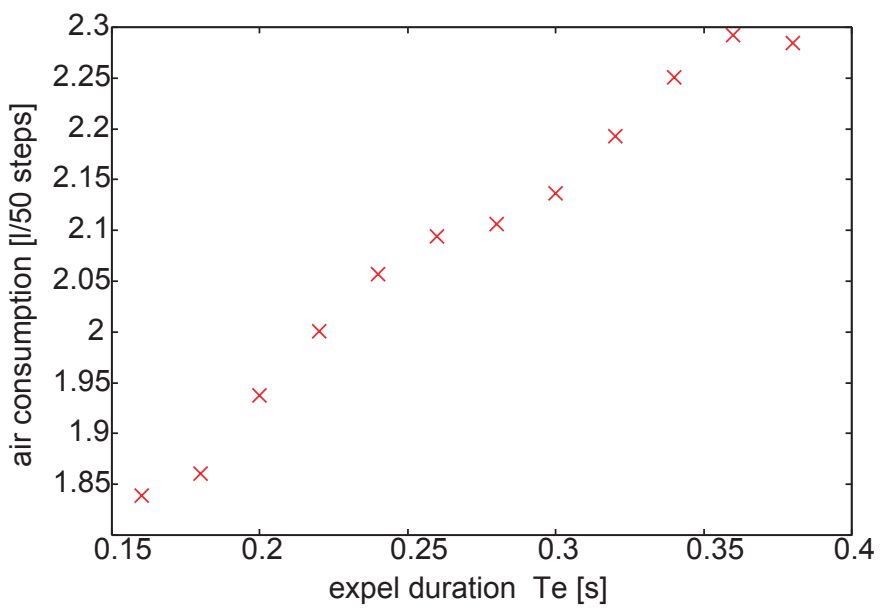

Fig. 11. relation between expel duration $S_{e}(k)$ and the air consumption 


\section{REFERENCES}

[1] R.Shiavi. Electromyographic patterns in adult locomotion: a comprehensive review. Journal of Rehabilitation Research and Development, Vol. 22, No. 3, pp. 85-98, 1985.

[2] S.Kajita, M.Morisawa, K.Harada, K.Kaneko, F.Kanehiro, K.Fujiwara, and H.Hirukawa. Biped walking pattern generator allowing auxiliary zmp control. In Proceedings of IEEE/RSJ International Conference on Intelligent Robots and Systems (IROS), pp. 2993-2999, 2006.

[3] S.Kajita, F.Kanehiro, K.Kaneko, K.Fujiwara, K.Yokoi, and H.Hirukawa. Biped walking pattern generation by a simple three-dimensional inverted pendulum model. Advanced Robotics, Vol. 17, No. 2, pp. 131-147, 2003.

[4] T. McGeer. Passive dynamic walking. International Journal of Robotics Research, Vol. 9, No. 2, pp. 62-82, 1990.

[5] F. Asano, M. Yamakita, N. Kawamichi, and Z. W. Luo. A novel gait generation for biped walking robots based on mechanical energy constraint. In Proceedings of the 2002 IEEE/RSJ International Conference on Intelligent Robots and Systems (IROS), pp. 2637-2644, 2002.

[6] C.T.Farley and O.González. Leg stiffness and stride frequency in human running. Journal of Biomechanics, Vol. 29, No. 2, pp. 181-186, 1996.

[7] R.Q.van der Linde. Passive bipedal walking with phasic muscle contraction. Biological Cybernetics, Vol. 81, No. 3, pp. 227-237, 1999.

[8] A. D. Kuo. Energetics of actively powered locomotion using the simples walking model. Journal of Biomechanical Engineering, Vol. 124, No. 1, pp. 113-120, February 2002.

[9] C. P. Chou and B. Hannaford. Measurement and modeling of mckibben pneumatic artificial muscles. IEEE Transactions on Robotics and Automation, Vol. 12, No. 1, pp. 90-102, 1996.

[10] R. Q. van der Linde. Design, analysis, and control of a low power joint for walking robots, by phasic activation of mckibben muscles. IEEE Transactions on Robotics and Automation, Vol. 15, No. 4, pp. 599-604, 1999.

[11] M. Wisse and J. van Frankenhuyzen. Design and construction of mike ; $2 \mathrm{~d}$ autonomous biped based on passeive dynamic walking. In 2 nd International Symposium on Adaptive Motion of Animals and Machined (AMAM), 2002.

[12] HITACHI Medical corporation web page. http://www.hitachimedical.co.jp/english/index.htm.

[13] Smc web page. http://www.smcworld.com. 